\title{
EL SESGO DE GÉNERO EN LA ASISTENCIA SANITARIA: DEFINICIÓN, CAUSAS Y CONSECUENCIAS EN LOS PACIENTES
}

Gender Bias in Healthcare: Definition, Causes and Consequences for Patients

Teresa Cabanillas-Montferrer ${ }^{1}$, Pepita Giménez-Bonafé ${ }^{1}$

Autor correspondencia: Pepita Giménez-Bonafé

Correo electrónico: pgimenez@ub.edu

1. Unidad de Fisiología, Departamento de Ciencias Fisiológicas, Facultad de Medicina y Ciencias de la Salud, Campus de Bellvitge, Universidad de Barcelona. (Hospitalet de LLobregat, España)

Recibido: 15/07/2021 Aceptado: 10/01/2022 


\section{RESUMEN}

OBJETIVO. El objetivo de esta revisión bibliográfica es aportar información sobre el origen y las consecuencias del sesgo de género en la actualidad, analizando las limitaciones que produce en la práctica clínica y proponiendo múltiples puntos que se deben abordar en un futuro para modificar el panorama actual. MATERIAL Y MÉTODO. Se ha realizado una búsqueda bibliográfica en bases de datos como Pubmed, Google Scholar y Dialnet, donde se han obtenido 241 registros no duplicados. Después de aplicar los criterios de exclusión, se reducen a 37, a partir de los cuales se identifican otros 21 artículos relevantes en bola de nieve. RESULTADOS. Se comenta brevemente el contenido de los 33 artículos que han sido relevantes para la investigación. DISCUSIÓN. El sesgo de género en la asistencia sanitaria es causado por su presencia en la producción y la difusión del conocimiento, dando lugar a un sesgo en el esfuerzo diagnóstico y terapéutico. Este hecho tiene consecuencias relacionadas con el conocimiento médico, el manejo clínico y la prestación de la asistencia sanitaria que repercuten en la salud de las mujeres, pero también de los hombres. Como limitaciones del estudio, cabe destacar que no se abordan la repercusión del sesgo de género en el colectivo sanitario, tanto en su formación como en su práctica laboral, ni en patologías concretas. CONCLUSIONES. Actualmente, se encuentran limitaciones relacionadas con el sesgo de género en el ámbito de la investigación, la difusión de conocimientos y la práctica clínica. Por ello, este continuará siendo un campo de investigación importante durante los próximos años, ya que la integración de la medicina de género es un elemento clave a tener en cuenta dentro de la medicina personalizada y la atención centrada en el paciente para alcanzar un enfoque holístico de los pacientes y las enfermedades.

Palabras clave: sesgo de género en medicina; perspectiva de género; diferencias de género; asistencia sanitaria; sexo y género; patología cardiovascular.

\section{ABSTRACT}

OBJECTIVES. The aim of this bibliographic review is to provide information on the origin and consequences of gender bias, analyzing the limitations that it produces in clinical practice and proposing multiple points that must be addressed in the future so that the current panorama can be modified. MATERIAL AND METHODS. A bibliographic search is performed on the Pubmed, Google Scholar and Dialnet databases, where 241 non-duplicate records are obtained. Following the application of the exclusion criteria, they are reduced to 37 articles, from which other 21 relevant items are identified by snowball sampling. RESULTS. The content of the 33 articles that have been relevant to the research is briefly commented. DISCUSSION. The gender bias in health care is caused by its presence in the production and dissemination of knowledge, leading to a bias in the diagnostic and therapeutic effort. This has consequences related to medical knowledge, clinical management and the provision of health care that have an impact on the health of women, but also of men. The highlighting study limitation is that the impact of gender bias in health professionals, both in their training and in their work practice, and in specific pathologies is not addressed. CONCLUSIONS. Currently, there are limitations related to gender bias in the field of research, dissemination of knowledge and clinical practice. Therefore, this will continue to be an important search field in the coming years, since the integration of gender medicine is a key item consider within personalized medicine and patient-centered care to achieve a holistic approach of patients and diseases.

Keywords: Gender Bias in Medicine; Gender Perspective; Gender Differences; Health Care; Sex and Gender; Cardiovascular Disease. 


\section{INTRODUCCIÓN}

Antes de empezar con la cuestión central de este artículo, cabe definir una serie de conceptos para poder llegar a una mejor comprensión de los aspectos tratados.

\section{Sexo y género}

Aunque en ocasiones son usados de manera indistinta, los conceptos de sexo y género son diferentes y no se pueden intercambiar. El sexo se define como el conjunto de atributos biológicos que se asocian con características físicas y fisiológicas, incluyendo cromosomas, expresión génica, función hormonal y anatomía reproductiva/sexual (CIHR, 2012). Pese a que la configuración cromosómica es compleja, se trata esta variable como dicotómica (hombre-mujer) (Ruiz-Cantero et al., 2019).

En cambio, el género es una construcción social dinámica basada en el sexo que hace referencia a los roles, comportamientos e identidades atribuibles a las personas femeninas, masculinas y de género diverso (CIHR, 2012). El género suele ser conceptualizado incorrectamente como un factor binario (femenino/masculino). En realidad, hay un espectro de identidades y expresiones de género que definen como los individuos se identifican y expresan su género (Heidari et al., 2016).

En las personas transgénero, la identidad de género es diferente del sexo que se les fue asignado al nacer. Hasta ahora, las personas transgénero han estado poco representadas en estudios clínicos, aunque esto está cambiando (Mauvais-Jarvis et al., 2020).

Las interacciones entre sexo y género influyen en la salud y el bienestar de diversas formas (Heidari et al., 2016). No siempre se puede distinguir el grado en que un fenómeno de salud es específicamente social o biológico (Risberg et al., 2003). Ambos son determinantes críticos de la salud (Hoffmann y Tarzian, 2001).

\section{Perspectiva y análisis de género}

La perspectiva de género se trata de una posición ideológica que toma en consideración y presta atención a las diferencias entre hombres y mujeres en cualquier actividad o ámbito dados (Europea, 1998). La aplicación de esta perspectiva centrándose en procesos sociales que describen y explican las diferencias y desigualdades entre mujeres y hombres se conoce como «análisis de género» (Ruiz Cantero, 2009). 
Una perspectiva de género en medicina define la necesidad de considerar la variable género como un factor clave al establecer y aplicar el conocimiento (Tasa-Vinyals, Mora Giral y Raich, 2015). Implica que se deben tener en cuenta las condiciones de vida, las posiciones en la sociedad y las expectativas sociales sobre la «feminidad»y la «masculinidad» juntamente con la biología (Risberg, Johansson y Hamberg, 2009).

\section{Sesgo de género}

El sesgo de género se define como «la diferencia en el tratamiento de ambos sexos con un mismo diagnóstico clínico, pudiendo tener consecuencias positivas, negativas o neutras para la salud de los mismos» (Ruiz-Cantero y Verdú-Delgado, 2004).

Hay dos posibles fuentes de sesgo de género en la asistencia sanitaria y la investigación. Una es la ceguera de género, es decir, asumir equidad entre hombres y mujeres cuando hay diferencias a tener en cuenta en biología, enfermedades, condiciones y experiencias vitales. La otra es asumir diferencias entre hombres y mujeres cuando no las hay, entendiendo como válidos los estereotipos dicotómicos asociados (Ruiz y Verbrugge, 1997). Ambas pueden dar situaciones de sesgo de medida y de selección tanto en situaciones epidemiológicas clínicas como sociales (Ruiz-Cantero et al., 2007). Todo esto ocasiona un sesgo de género cognitivo, social y constitucional (Vázquez-Santiago y Garrido Peña, 2016) que da lugar a conocimientos sesgados, condicionando una práctica profesional discriminatoria hacia un sexo o el otro en los servicios sanitarios, así como en la promoción de la salud y los servicios preventivos y las agendas políticas (Ruiz-Cantero et al., 2007).

Además de sesgo de género, se ha observado que el género se interrelaciona con otros condicionantes de la estratificación social produciendo cada uno de ellos un sesgo propio que puede interaccionar con los demás (Esteban Galarza, 2006).

Una vez definidos estos conceptos, es necesario explicar brevemente el contexto histórico que rodea la cuestión central de esta revisión. Los problemas de salud de las mujeres se han observado desde una mirada androcéntrica a lo largo de la historia, es decir, no han tenido visibilidad a causa de un sesgo inconsciente de que los problemas de hombres y mujeres son similares y que es posible extrapolarlos (Valls-Llobet, 2001). Además, la salud femenina se ha visto casi exclusivamente en términos de salud reproductiva. Este concepto recibe el nombre de esencialismo biológico, donde se caracteriza a las mujeres como un grupo de riesgo único y universal, el cual es definido 
basándose en su capacidad biológica reproductiva (Ruiz Cantero, 2009; Chilet-Rosell, 2014). Estos hechos pueden propiciar la aparición de sesgo de género en la práctica clínica, teniendo repercusiones positivas, negativas o neutras para la salud tanto de las mujeres como de los hombres.

Así, pues, mediante la síntesis de la evidencia científica disponible, el objetivo principal de esta revisión bibliográfica es aportar información sobre el origen y las consecuencias del sesgo de género en la actualidad, analizando las limitaciones que produce en la práctica clínica y proponiendo múltiples puntos que se deben abordar en un futuro para modificar el panorama actual.

\section{MATERIAL Y MÉTODO}

Se ha realizado una búsqueda bibliográfica inicial de carácter narrativo sin límite de fecha inferior y hasta el año 2020 incluido, utilizando las bases de datos Pubmed, Google Scholar y Dialnet, ya que eran aquellas que aportaban un mayor número de resultados relacionados con el tema revisado. Además, se han consultado algunas publicaciones de estamentos oficiales.

La búsqueda se ha realizado principalmente a partir de la combinación de palabras clave relacionadas con el tema central de la revisión. Concretamente, las búsquedas principales se encuentran recogidas en la Tabla 1:

Tabla 1. Búsquedas principales de la revisión bibliográfica

\begin{tabular}{|c|c|c|}
\hline $\begin{array}{l}\text { Base de datos } \\
\text { utilizada }\end{array}$ & Búsqueda realizada & $\begin{array}{l}\text { Núm. de } \\
\text { resultados }\end{array}$ \\
\hline Pubmed & 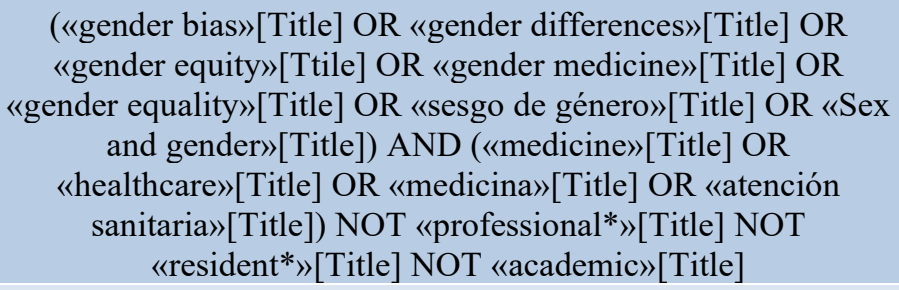 & 201 \\
\hline Google Scholar & allintitle: gender bias medicine «gender bias» & 48 \\
\hline Dialnet & «sesgo de género» AND medicina & 15 \\
\hline
\end{tabular}

A partir de estos resultados, debido a que el sesgo de género en salud es un campo muy amplio con múltiples dimensiones a analizar, se han aplicado unos criterios de selección, incluyendo solo aquellos artículos escritos en inglés o castellano que aportan una visión centrada en el paciente y global sobre la temática de la revisión. En 
consecuencia, se han descartado aquellos artículos que abordan la repercusión del sesgo de género en los diferentes grupos de profesionales sanitarios y también aquellos que se focalizan en una patología concreta. Los criterios de exclusión que se han definido son los siguientes:

- La no relación con la temática de la revisión.

- La visión no global, con focalización en una patología concreta.

- El enfoque en el profesional y no en el paciente.

- La no posibilidad de acceso.

- El no uso de inglés o castellano como lenguas de escritura.

El proceso de búsqueda y selección inicial de la revisión bibliográfica se muestra de manera resumida en la Figura 1:

Figura 1. Diagrama de flujo PRISMA

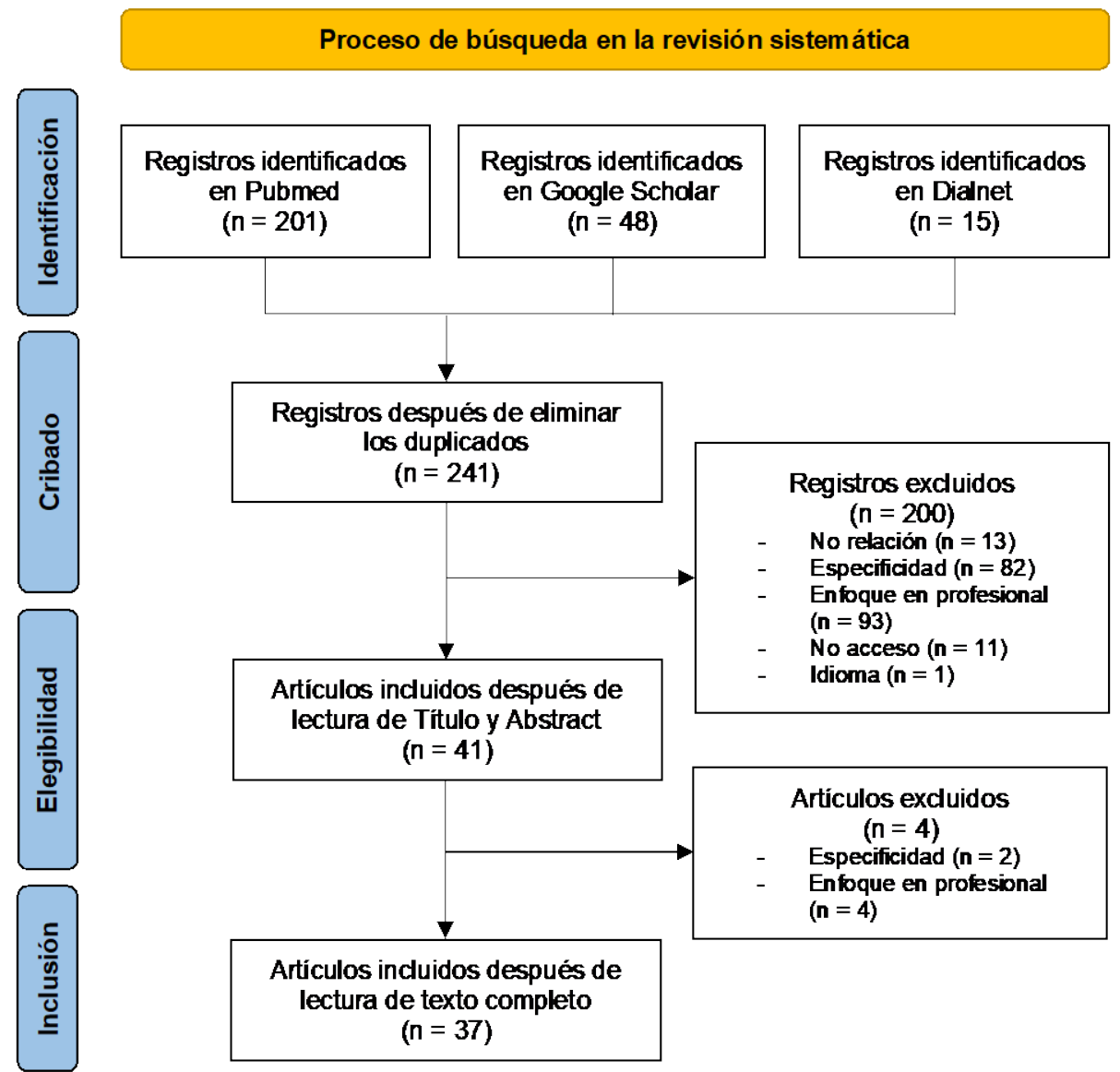


Finalmente, 12 de los artículos incluidos al final del proceso resultan ser de relevancia para los subtemas tratados en esta revisión bibliográfica. Se decide ampliar la búsqueda y se identifican en bola de nieve los otros 21 escritos revisados, los cuales se encuentran citados en varios de los escritos identificados, pero no se han obtenido como resultado en las búsquedas iniciales hechas en bases de datos.

La búsqueda ha sido de elevada dificultad, a causa de que la gran mayoría de escritos publicados no son de alta evidencia científica como revisiones sistemáticas, metaanálisis o guidelines. Esto ha obligado a la lectura de un gran número de artículos para acabar extrayendo aquellos más significativos dentro del tema seleccionado.

\section{RESULTADOS}

Entre los artículos revisados que han sido relevantes para la investigación se encuentran 11 revisiones bibliográficas y 21 artículos originales. Además, se ha incluido una publicación de un estamento oficial. A continuación, se muestra una tabla donde se expone brevemente el contenido de cada uno de ellos:

\begin{tabular}{|c|c|c|c|}
\hline Artículo & Autores & $\begin{array}{c}\text { Año de } \\
\text { publicación }\end{array}$ & Contenido \\
\hline $\begin{array}{l}\text { What a difference sex } \\
\text { and gender make }\end{array}$ & $\begin{array}{l}\text { CIHR Institute of } \\
\text { Gender and } \\
\text { Health }\end{array}$ & 2012 & $\begin{array}{l}\text { Se definen los conceptos de sexo y género. } \\
\text { Se exponen ejemplos donde se ilustra cómo los } \\
\text { procesos y resultados en la investigación de la salud } \\
\text { pueden ser diferentes si se tienen en cuenta las } \\
\text { influencias de género y sexo, concluyendo que se } \\
\text { deberían integrar de rutina en esta. }\end{array}$ \\
\hline $\begin{array}{l}\text { Gender agenda in } \\
\text { health sciences } \\
\text { education: } \\
\text { international } \\
\text { experiences to reduce } \\
\text { time in Spain }\end{array}$ & $\begin{array}{l}\text { Ruiz-Cantero, M. } \\
\text { T., Tomás-Aznar, } \\
\text { C., Rodríguez- } \\
\text { Jaume M. J., } \\
\text { Pérez-Sedeño, E., } \\
\text { Gasch-Gallén, Á. }\end{array}$ & 2019 & $\begin{array}{l}\text { Se analizan experiencias de la incorporación de la } \\
\text { perspectiva de género en titulaciones de ciencias de la } \\
\text { salud en el ámbito internacional, llegando a la } \\
\text { conclusión de que las claves para ello radican en los } \\
\text { niveles políticos, organizativos y de cambio cultural, y } \\
\text { en el profesorado, siendo imprescindible el apoyo } \\
\text { institucional para poder asegurar su sostenibilidad. }\end{array}$ \\
\hline $\begin{array}{l}\text { Sex and Gender Equity } \\
\text { in Research: rationale } \\
\text { for the SAGER } \\
\text { guidelines and } \\
\text { recommended use }\end{array}$ & $\begin{array}{l}\text { Heidari, S., } \\
\text { Babor, T. F., De } \\
\text { Castro, P., Tort, } \\
\text { S., Curno, M. }\end{array}$ & 2016 & $\begin{array}{l}\text { Se describen los fundamentos de un conjunto } \\
\text { internacional de directrices para fomentar un enfoque } \\
\text { más sistemático que tenga en consideración el sexo y el } \\
\text { género en el diseño de estudios, el análisis de datos y la } \\
\text { interpretación de resultados. }\end{array}$ \\
\hline $\begin{array}{l}\text { Sex and gender: } \\
\text { modifiers of health, } \\
\text { disease, and medicine }\end{array}$ & $\begin{array}{l}\text { Mauvais-Jarvis, } \\
\text { F., Bairey Merz, } \\
\text { N., Barnes, P. J., } \\
\text { Brinton, R. D., } \\
\text { Carrero, J. J., } \\
\text { DeMeo, D. L.,et } \\
\text { al. }\end{array}$ & 2020 & $\begin{array}{l}\text { Se describe la influencia de la genética, la epigenética y } \\
\text { las hormonas del sexo biológico en la fisiología y las } \\
\text { enfermedades. Se expone cómo el constructo social del } \\
\text { género afecta al comportamiento de la comunidad, los } \\
\text { médicos y los pacientes en la asistencia sanitaria e } \\
\text { interactúa con la patobiología. }\end{array}$ \\
\hline
\end{tabular}




\begin{tabular}{|c|c|c|c|}
\hline $\begin{array}{l}\text { Gender in medicine - } \\
\text { An issue for women } \\
\text { only? A survey of } \\
\text { physician teachers' } \\
\text { gender attitudes }\end{array}$ & $\begin{array}{l}\text { Risberg, G., } \\
\text { Johansson, E. E., } \\
\text { Westman, G., } \\
\text { Hamberg, K. }\end{array}$ & 2003 & $\begin{array}{l}\text { Se analiza el grado de importancia que le otorgan al } \\
\text { género en las relaciones profesionales en el ámbito de } \\
\text { la salud un grupo de médicos docentes de una } \\
\text { universidad sueca, concluyendo que, para implementar } \\
\text { la perspectiva de género en la educación, se necesita la } \\
\text { implicación de los profesores, tanto de sexo femenino } \\
\text { como masculino. }\end{array}$ \\
\hline $\begin{array}{l}\text { The Girl Who Cried } \\
\text { Pain: A Bias Against } \\
\text { Women in the } \\
\text { Treatment of Pain }\end{array}$ & $\begin{array}{l}\text { Hoffmann, D., } \\
\text { Tarzian, A. }\end{array}$ & 2001 & $\begin{array}{l}\text { Se realiza una revisión sobre la experiencia del dolor en } \\
\text { hombres y mujeres, tanto su percepción como su } \\
\text { diagnóstico y tratamiento por parte del colectivo } \\
\text { sanitario. Se sugieren distintos métodos para mejorar el } \\
\text { manejo del dolor tanto en hombres como en mujeres. }\end{array}$ \\
\hline $\begin{array}{l}100 \text { palabras para la } \\
\text { igualdad. Glosario de } \\
\text { términos relativos a la } \\
\text { igualdad entre mujeres } \\
\text { y hombres }\end{array}$ & $\begin{array}{l}\text { Comisión } \\
\text { Europea }\end{array}$ & 1998 & $\begin{array}{l}\text { Glosario donde se reúnen todos los términos utilizados } \\
\text { comúnmente en el campo de la política de la igualdad } \\
\text { de oportunidades, contribuyendo así a crear un lenguaje } \\
\text { común en Europa para quienes trabajan en este ámbito. }\end{array}$ \\
\hline $\begin{array}{l}\text { Sesgos de género en la } \\
\text { atención sanitaria }\end{array}$ & $\begin{array}{c}\text { Ruiz Cantero, M. } \\
\text { T. }\end{array}$ & 2009 & $\begin{array}{c}\text { Se realiza una revisión de las epistemologías feministas } \\
\text { y se analizan los sesgos que se producen en la atención } \\
\text { a las mujeres dentro del sistema sanitario. }\end{array}$ \\
\hline $\begin{array}{l}\text { Sesgo de género en } \\
\text { medicina: concepto y } \\
\text { estado de la cuestión }\end{array}$ & $\begin{array}{l}\text { Tasa-Vinyals, E., } \\
\text { Mora Giral, M., } \\
\text { Raich, R. }\end{array}$ & 2015 & $\begin{array}{l}\text { Se exponen y discuten la génesis y las consecuencias } \\
\text { del androcentrismo médico. Se discuten también los } \\
\text { conceptos de perspectiva y sensibilidad de género en la } \\
\text { investigación biomédica, epidemiológica y clínica para } \\
\text { alcanzar un conocimiento médico más preciso e } \\
\text { inclusivo y conseguir un sistema de salud más justo y } \\
\text { equitativo. }\end{array}$ \\
\hline $\begin{array}{l}\text { A theoretical model for } \\
\text { analysing gender bias } \\
\text { in medicine }\end{array}$ & $\begin{array}{l}\text { Risberg, G., } \\
\text { Johansson, E. E., } \\
\text { Hamberg, K. }\end{array}$ & 2009 & $\begin{array}{l}\text { Se desarrolla un modelo para entender cómo se produce } \\
\text { el sesgo de género en medicina y se discute su utilidad } \\
\text { en los esfuerzos para evitar que esto pase. }\end{array}$ \\
\hline $\begin{array}{l}\text { Sesgo de género en el } \\
\text { esfuerzo terapéutico }\end{array}$ & $\begin{array}{l}\text { Ruiz-Cantero, M. } \\
\text { T., Verdú- } \\
\text { Delgado, M. }\end{array}$ & 2004 & $\begin{array}{l}\text { Se muestra una aproximación a la evidencia de sesgo } \\
\text { de género en el esfuerzo terapéutico en España, } \\
\text { concluyendo que falta información sistemática sobre } \\
\text { este. }\end{array}$ \\
\hline $\begin{array}{c}\text { A two-way view of } \\
\text { gender bias in medicine }\end{array}$ & $\begin{array}{l}\text { Ruiz, M. T., } \\
\text { Verbrugge, L. M. }\end{array}$ & 1997 & $\begin{array}{c}\text { Se exponen los orígenes y las consecuencias del sesgo } \\
\text { de género, y las limitaciones y desafíos que supone en } \\
\text { la práctica clínica. }\end{array}$ \\
\hline $\begin{array}{l}\text { A framework to analyse } \\
\text { gender bias in } \\
\text { epidemiological } \\
\text { research }\end{array}$ & $\begin{array}{l}\text { Ruiz-Cantero, M. } \\
\text { T., Vives-Cases, } \\
\text { C., Artazcoz, L., } \\
\text { Delgado, A., } \\
\text { Calvente, } \\
\text { MDMG, Miqueo, } \\
\text { C. }\end{array}$ & 2007 & $\begin{array}{l}\text { Se propone un marco para analizar el sesgo de género } \\
\text { en el diseño y análisis de las investigaciones realizadas } \\
\text { sobre problemas de salud de la mujer. }\end{array}$ \\
\hline $\begin{array}{l}\text { El enfoque de género en } \\
\text { las necesidades de } \\
\text { atención sociosanitaria }\end{array}$ & $\begin{array}{l}\text { Vázquez- } \\
\text { Santiago, S., } \\
\text { Garrido Peña, F. }\end{array}$ & 2016 & $\begin{array}{c}\text { Se analiza el sesgo de género en la atención sanitaria } \\
\text { como una parte relevante dentro de los determinantes } \\
\text { sociales de la salud, sugiriéndose varias medidas para } \\
\text { minimizar su impacto. }\end{array}$ \\
\hline $\begin{array}{l}\text { El estudio de la salud y } \\
\text { el género: las ventajas } \\
\text { de un enfoque } \\
\text { antropológico y } \\
\text { feminista }\end{array}$ & $\begin{array}{c}\text { Esteban Galarza, } \\
\text { M. }\end{array}$ & 2006 & $\begin{array}{l}\text { Se muestran las ventajas de un enfoque antropológico y } \\
\text { feminista para estudiar los procesos de } \\
\text { salud/enfermedad/atención referidos exclusivamente o } \\
\text { no a las relaciones de género. }\end{array}$ \\
\hline
\end{tabular}




\begin{tabular}{|c|c|c|c|}
\hline $\begin{array}{l}\text { Desigualdades de } \\
\text { género en salud pública }\end{array}$ & Valls-Llobet, C. & 2001 & $\begin{array}{l}\text { Se propone el estudio de las desigualdades de género en } \\
\text { el terreno de la salud desde una visión de los problemas } \\
\text { de salud basada en lo biológico, psicológico y social. } \\
\text { Se desarrolla el concepto de mirada androcéntrica y se } \\
\text { exponen las consecuencias del sesgo de género y las } \\
\text { desigualdades sociales, planteando finalmente varios } \\
\text { retos en relación con el sesgo de género. }\end{array}$ \\
\hline $\begin{array}{l}\text { Gender bias in clinical } \\
\text { research, } \\
\text { pharmaceutical } \\
\text { marketing, and the } \\
\text { prescription of drugs }\end{array}$ & Chilet-Rosell, E. & 2014 & $\begin{array}{l}\text { A partir de la realización de múltiples estudios, se } \\
\text { concluye que la legitimidad de la medicina se encuentra } \\
\text { limitada, ya que no se basa en la mejor evidencia } \\
\text { posible. Además, se observan desigualdades entre } \\
\text { hombres y mujeres que no se deben meramente a } \\
\text { diferencias biológicas, sino que derivan de las } \\
\text { diferencias sociales que existen entre ambos sexos. }\end{array}$ \\
\hline $\begin{array}{l}\text { Clinical Trials and } \\
\text { Experimental Science, } \\
\text { Bias Against Women in }\end{array}$ & Rosser, S. V. & 2015 & $\begin{array}{l}\text { Se explican algunas de las diferentes etapas de } \\
\text { aplicación del método científico en los ensayos clínicos } \\
\text { donde se puede ver reflejado el sesgo de género. }\end{array}$ \\
\hline $\begin{array}{l}\text { Wanted: Women in } \\
\text { clinical trials }\end{array}$ & Simon, V. & 2015 & $\begin{array}{c}\text { Se realiza una descripción breve de la evolución y el } \\
\text { papel actual de las mujeres en los ensayos clínicos, } \\
\text { destacando la importancia de incluir el sexo en el } \\
\text { análisis de los resultados. }\end{array}$ \\
\hline $\begin{array}{l}\text { Gender equality in } \\
\text { science, medicine, and } \\
\text { global health: where } \\
\text { are we at and why does } \\
\text { it matter? }\end{array}$ & $\begin{array}{l}\text { Shannon, G., } \\
\text { Jansen, M., } \\
\text { Williams, K., } \\
\text { Cáceres, C., } \\
\text { Motta, A., } \\
\text { Odhiambo, A. }\end{array}$ & 2019 & $\begin{array}{l}\text { Se proporciona evidencia de por qué la igualdad de } \\
\text { género en la ciencia y la medicina es importante para la } \\
\text { salud y los resultados relacionados con esta. }\end{array}$ \\
\hline $\begin{array}{c}\text { Quality of } \\
\text { pharmaceutical } \\
\text { advertising and gender } \\
\text { bias in medical journals } \\
\text { (1998-2008): A review } \\
\text { of the scientific } \\
\text { literature }\end{array}$ & $\begin{array}{l}\text { Cambronero Saiz, } \\
\text { B., Ruiz Cantero, } \\
\text { M. T., Papí } \\
\text { Gálvez, N. }\end{array}$ & 2012 & $\begin{array}{c}\text { Se revisa la literatura científica sobre publicidad } \\
\text { farmacéutica dirigida a profesionales de la salud para } \\
\text { determinar si el sesgo de género ha disminuido y la } \\
\text { calidad de la información en la publicidad farmacéutica } \\
\text { ha mejorado con el tiempo. }\end{array}$ \\
\hline $\begin{array}{l}\text { What is missing in } \\
\text { measures of Women's } \\
\text { Empowerment? }\end{array}$ & Cueva Beteta, H. & 2006 & $\begin{array}{l}\text { Se realiza una evaluación de los diferentes indicadores } \\
\text { que se deben tener en cuenta para medir el } \\
\text { empoderamiento de género, entendiendo este como un } \\
\text { proceso y no como un resultado. }\end{array}$ \\
\hline $\begin{array}{l}\text { Gender bias in clinical } \\
\text { trials: Do double } \\
\text { standards still apply? }\end{array}$ & $\begin{array}{l}\text { Ramasubbu, K., } \\
\text { Gurm, H., } \\
\text { Litaker, D. }\end{array}$ & 2001 & $\begin{array}{l}\text { Se revisan múltiples ensayos clínicos, valorando el } \\
\text { porcentaje de mujeres inscritas en ellos y el análisis de } \\
\text { datos específicos de género, obteniendo como resultado } \\
\text { una inscripción de género poco equilibrada y una baja } \\
\text { promoción del análisis mencionado. }\end{array}$ \\
\hline $\begin{array}{l}\text { Global health burden } \\
\text { and needs of } \\
\text { transgender } \\
\text { populations: a review }\end{array}$ & $\begin{array}{l}\text { Reisner, S. L., } \\
\text { Poteat, T., } \\
\text { Keatley, J. A., } \\
\text { Cabral, M., } \\
\text { Mothopeng, T., } \\
\text { Dunham, E. }\end{array}$ & 2016 & $\begin{array}{l}\text { Se revisan múltiples artículos sobre indicadores } \\
\text { negativos de salud en las personas transgénero, } \\
\text { reportándose falta de datos y conocimiento sobre ellas } \\
\text { en muchas áreas de salud. }\end{array}$ \\
\hline $\begin{array}{l}\text { Women's Health } \\
\text { Education Initiatives: } \\
\text { Why Have They } \\
\text { Stalled? }\end{array}$ & Henrich, J. B. & 2004 & $\begin{array}{c}\text { Se analiza la situación actual con respecto a la } \\
\text { integración de conocimientos específicos de la salud de } \\
\text { la mujer en el plan de estudio de las universidades de } \\
\text { medicina. }\end{array}$ \\
\hline
\end{tabular}




\begin{tabular}{|c|c|c|c|}
\hline $\begin{array}{c}\text { Gender bias in } \\
\text { medicine }\end{array}$ & Hamberg, K. & 2008 & $\begin{array}{c}\text { Se examina el fenómeno del sesgo de género en la } \\
\text { medicina, presentándose conceptos y modelos útiles } \\
\text { para la comprensión del sesgo y describiéndose algunas } \\
\text { áreas de interés para futuras investigaciones. }\end{array}$ \\
\hline $\begin{array}{l}\text { Reflexiones acerca de } \\
\text { las dificultades en la } \\
\text { integración del enfoque } \\
\text { de género en la } \\
\text { investigación en salud }\end{array}$ & Aldasoro, E. & 2017 & $\begin{array}{l}\text { Se reflexiona sobre algunas dificultades y barreras } \\
\text { identificadas para la incorporación del enfoque de } \\
\text { género en la investigación en salud. }\end{array}$ \\
\hline $\begin{array}{l}\text { Why are sex and } \\
\text { gender important to } \\
\text { basic physiology and } \\
\text { translational and } \\
\text { individualized } \\
\text { medicine? }\end{array}$ & Miller, V. M. & 2014 & $\begin{array}{l}\text { Se aclara la definición de sexo y género y se revisa la } \\
\text { evidencia que muestra cómo el sexo y el género } \\
\text { interactúan entre sí para influir en la etiología, la } \\
\text { presentación de la enfermedad y los resultados del } \\
\text { tratamiento. Se presentan estrategias para mejorar la } \\
\text { inclusión de seres humanos femeninos y masculinos en } \\
\text { estudios preclínicos y clínicos, y se discute la } \\
\text { importancia de incorporar conceptos de sexo y género } \\
\text { en los planes de estudios de posgrado y médicos. }\end{array}$ \\
\hline $\begin{array}{l}\text { Las desigualdades de } \\
\text { género en la ciencia: } \\
\text { Gaceta Sanitaria da un } \\
\text { paso adelante }\end{array}$ & $\begin{array}{l}\text { Borrell, C., } \\
\text { Vives-Cases, C., } \\
\text { Domínguez- } \\
\text { Berjón, M. F., } \\
\text { Álvarez-Dardet, } \\
\text { C. }\end{array}$ & 2015 & $\begin{array}{l}\text { Se contextualiza y presenta una iniciativa para } \\
\text { responder a las desigualdades de género en las } \\
\text { instituciones y estructuras relacionadas con la ciencia y } \\
\text { la producción científica, fomentando así la igualdad de } \\
\text { género. }\end{array}$ \\
\hline $\begin{array}{l}\text { Better science with sex } \\
\text { and gender: } \\
\text { Facilitating the use of a } \\
\text { sex and gender-based } \\
\text { analysis in health } \\
\text { research }\end{array}$ & $\begin{array}{l}\text { Johnson, J. L., } \\
\text { Greaves, L., } \\
\text { Repta, R. }\end{array}$ & 2009 & $\begin{array}{c}\text { Se enfatiza la necesidad de una atención continua al } \\
\text { sexo y el género en la investigación en salud, } \\
\text { proporcionando información sobre género y sexo, } \\
\text { análisis de género y múltiples enfoques para incorporar } \\
\text { estos conceptos a varias etapas del proceso de } \\
\text { investigación. }\end{array}$ \\
\hline $\begin{array}{c}\text { Sex-Specific } \\
\text { Considerations in } \\
\text { Guidelines Generation } \\
\text { and Application }\end{array}$ & $\begin{array}{l}\text { Tannenbaum, C., } \\
\text { Norris, C. M., } \\
\text { McMurtry, M. S. }\end{array}$ & 2019 & $\begin{array}{l}\text { Se revisa cómo los comités de guías de práctica clínica } \\
\text { pueden incorporar un marco estructurado para } \\
\text { determinar la calidad de la evidencia en las } \\
\text { evaluaciones específicas por sexo. Se discuten los } \\
\text { desafíos en curso y se brindan soluciones sobre cómo } \\
\text { desglosar la evidencia, cómo evaluar el sesgo, cómo } \\
\text { mejorar las estrategias de búsqueda y qué hacer cuando } \\
\text { los datos son insuficientes para hacer recomendaciones } \\
\text { específicas por sexo. }\end{array}$ \\
\hline $\begin{array}{l}\text { Sex and gender } \\
\text { differences in health. } \\
\text { Science \& Society } \\
\text { Series on Sex and } \\
\text { Science }\end{array}$ & $\begin{array}{c}\text { Regitz-Zagrosek, } \\
\text { V. }\end{array}$ & 2012 & $\begin{array}{l}\text { Se analizan las diferencias presentes entre sexos y } \\
\text { géneros y cómo estas se deben tener en cuenta en el } \\
\text { diagnóstico, el tratamiento y el manejo clínico de las } \\
\text { patologías para así mejorar la calidad de la atención } \\
\text { sanitaria. }\end{array}$ \\
\hline $\begin{array}{l}\text { «Bridging the Gap» } \\
\text { Everything that Could } \\
\text { Have Been Avoided If } \\
\text { We Had Applied } \\
\text { Gender Medicine, } \\
\text { Pharmacogenetics and } \\
\text { Personalized Medicine } \\
\text { in the Gender-Omics } \\
\text { and Sex-Omics Era }\end{array}$ & $\begin{array}{l}\text { Gemmati, D., } \\
\text { Varani, K., } \\
\text { Bramanti, B., } \\
\text { Piva, R., } \\
\text { Bonaccorsi, G., } \\
\text { Trentini, A. }\end{array}$ & 2020 & $\begin{array}{l}\text { Se exponen múltiples conocimientos sobre la medicina } \\
\text { de género, abarcando desde las posturas moleculares } \\
\text { hasta las sociales y psicológicas. Se realiza la } \\
\text { descripción y discusión crítica de algunos temas clave } \\
\text { multidisciplinarios considerados como paradigmáticos } \\
\text { de las diferencias de sexo/género y las desigualdades de } \\
\text { sexo/género. }\end{array}$ \\
\hline
\end{tabular}




\section{DISCUSIÓN}

\section{Causas del sesgo de género en la asistencia sanitaria}

De acuerdo con Chilet-Rosell (2014) y tal como muestra la Figura 2, el sesgo de género en la asistencia sanitaria es el producto de su arraigo este en la producción y difusión del conocimiento.

Figura 2. Interconexión entre la producción del conocimiento, su difusión y la asistencia sanitaria (modificado de Chilet-Rosell, 2014).

\subsection{Sesgo de género en la producción del conocimiento}

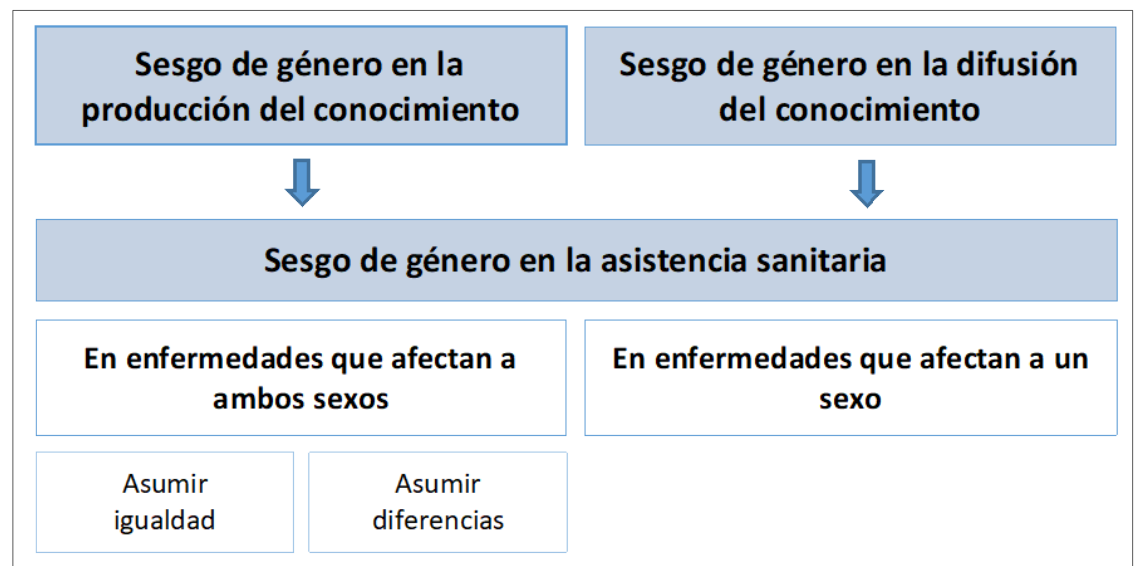

Tal y como se expone en Rosser (2015), el sesgo de género no intencionado se puede reflejar en al menos cuatro etapas de aplicación del método científico:

\section{Exclusión de las mujeres como sujetos experimentales y de diseño}

Históricamente, la mayoría de los ensayos clínicos se han realizado incluyendo mayoritariamente hombres y asumiendo que los resultados pueden ser automáticamente aplicados al sexo femenino, como si este se hubiera estudiado también. Se extrapola la presentación clínica vista en el sexo masculino al femenino y se asume que los factores de riesgo y protectores son los mismos. Por otro lado, factores de riesgo que son cruciales para las mujeres, como los debidos a la sobrecarga desigual en la atención a la familia y al trabajo doméstico, aún tienen que ser plenamente investigados (Valls-Llobet, 2001). Aun así, gracias a algunos cambios en las leyes, en la FDA y el NIH, la participación de las mujeres en los ensayos clínicos se encuentra en aumento (Ruiz Cantero, 2009).

A pesar de la existencia de diferencias entre sexos en farmacocinética y farmacodinámica, la dificultad superior en incorporar a las mujeres a los ensayos, su alta tasa de abandono durante el seguimiento, los efectos de confusión provocados por los 
cambios hormonales y las interacciones con otros tratamientos concomitantes son algunos de los argumentos para su exclusión, pero justamente estas razones hacen recomendable su inclusión (Ruiz-Cantero y Verdú-Delgado, 2004).

Uno de los principales argumentos para no elaborar un análisis por sexo o un análisis de las interacciones hormonales es el coste que supone utilizar un tamaño muestral suficiente para detectar diferencias. No obstante, retirar un fármaco del mercado a causa de la presencia superior de efectos adversos en las mujeres, posiblemente en relación con la falta de información sobre esta parte de la población, es todavía más costoso (Simon, 2005).

\section{Elección y definición de problemas a estudiar}

La variable sexo no siempre se incorpora en el análisis del trabajo ni la perspectiva de género en la hipótesis principal, el análisis y la interpretación de la información (RuizCantero y Verdú-Delgado, 2004). Algunos de los ítems que preocupan más a las mujeres reciben menos financiación y estudio. El enfoque excesivo en la investigación con sujetos de sexo masculino y la definición de ciertas patologías como «masculinas» da lugar a un infradiagnóstico y subtratamiento de estas en las mujeres. Este hecho también se puede observar a la inversa en algunos casos. El género del investigador puede influir en la elección del problema a resolver y, según los datos de la UN Educational, Scientific and Cultural Organization's Women in Science, menos del 30\% de los investigadores del mundo son mujeres, aunque esta proporción se encuentra en aumento (Shannon et al., 2019).

La asunción de un mejor estado de salud de las mujeres a causa de las mayores tasas de mortalidad y la menor esperanza de vida del sexo masculino lleva a un mayor interés por la investigación de las enfermedades que causan una alta mortalidad entre hombres, reforzando los mitos de que las enfermedades crónicas en general son más graves, frecuentes y fatales en hombres e ignorando el hecho de la mayor prevalencia en mujeres de condiciones crónicas no mortales (Ruiz y Verbrugge, 1997).

\section{Métodos y enfoques utilizados en la recopilación de datos}

El uso del hombre blanco, de mediana edad, heterosexual como el «sujeto experimental básico» no solo ignora el hecho de que las mujeres pueden responder de un modo diferente a la variable probada, sino que también puede conducir a modelos menos precisos incluso para muchos hombres. 


\section{Teorías y conclusiones extraídas de los datos}

Estas pueden extenderse inapropiadamente más allá de la población estudiada o aplicarse incorrectamente a otras poblaciones, reforzando así, con frecuencia, el sesgo. Concluir, a partir de datos de investigación, que solo puede resultar un diseño posible puede reflejar el sesgo y limitar la exploración de otras posibles interpretaciones de datos.

\subsection{Sesgo de género en la difusión del conocimiento}

Tal y como se explica en Borrell et al. (2015), el sesgo de género en la difusión de conocimiento viene condicionado por las desigualdades de género en las estructuras de poder en la ciencia y en las revistas médicas. Las mujeres se encuentran infrarepresentadas en las posiciones de más rango académico y en los órganos de decisión sobre la ciencia. Las revistas científicas son un importante canal de comunicación de los avances de la ciencia y la presencia de mujeres en los órganos de decisión de estas publicaciones es menor.

Por otro lado, hay desigualdades de género en la producción de documentos científicos y un análisis bibliométrico mundial muestra que en casi todos los países las mujeres son autoras en menor proporción y, además, los manuscritos firmados por mujeres atraen menos citas. Sin embargo, el nombre de autoras está aumentando a lo largo de los años. Otro punto que cabe destacar es la insensibilidad de género en el sistema de recuperación de información Medline/Pubmed y Cochrane. El proceso de selección bibliográfica para cualquier investigación médica y el debate de perspectiva de género puede verse afectado por el androcentrismo prevalente en la ciencia tradicional (RuizCantero et al., 2007).

Por último, la publicidad farmacéutica puede contribuir a reforzar la percepción de que ciertas enfermedades se asocian con el sexo más frecuentemente representado en esta. Hay una tendencia constante a encarnar imágenes estereotipadas de ambos sexos (Cambronero Saiz, Ruiz Cantero y Papí Gálvez, 2012).

\subsection{Interconexión entre producción del conocimiento, difusión y atención médica}

La definición de sesgo de género en la práctica clínica atribuye la responsabilidad exclusiva a los profesionales sanitarios. No obstante, los profesionales diagnostican y tratan de acuerdo con la formación recibida y la información que tienen disponible. Por 
lo tanto, se puede deducir que el sesgo de género en la práctica clínica es causado por errores en la investigación a consecuencia de la manca de consciencia de género, es decir, el sesgo de género en la atención sanitaria es, en gran parte, resultado del sesgo de género en la generación de conocimiento. Además, es necesario considerar que el márquetin se ha convertido en uno de los filtros más importantes del conocimiento médico, ayudando a fortalecer erróneamente la percepción de que ciertas enfermedades son más frecuentes en un sexo que en el otro y contribuyendo a la medicalización de los procesos vitales de las mujeres (Chilet-Rosell, 2014). Al ser la investigación la base de la práctica clínica y esta última el sentido y la razón de ser de la primera, los fenómenos de sesgo existentes en ambas no pueden sino retroalimentarse y multiplicar sus efectos (Tasa-Vinyals, Mora Giral y Raich, 2015).

\section{Principales dimensiones del sesgo de género en la asistencia sanitaria}

\subsection{En el esfuerzo diagnóstico}

Tal y como expone en Ruiz Cantero (2009) y se refleja en la Figura 3, los pacientes acuden a la demanda asistencial con una serie de características propias de su enfermedad y personales, así como con una percepción y expresión de los síntomas y una valoración de la gravedad determinada.

Figura 3. Condicionantes del esfuerzo diagnóstico y terapéutico (Modificado de Ruiz Cantero [2009]).

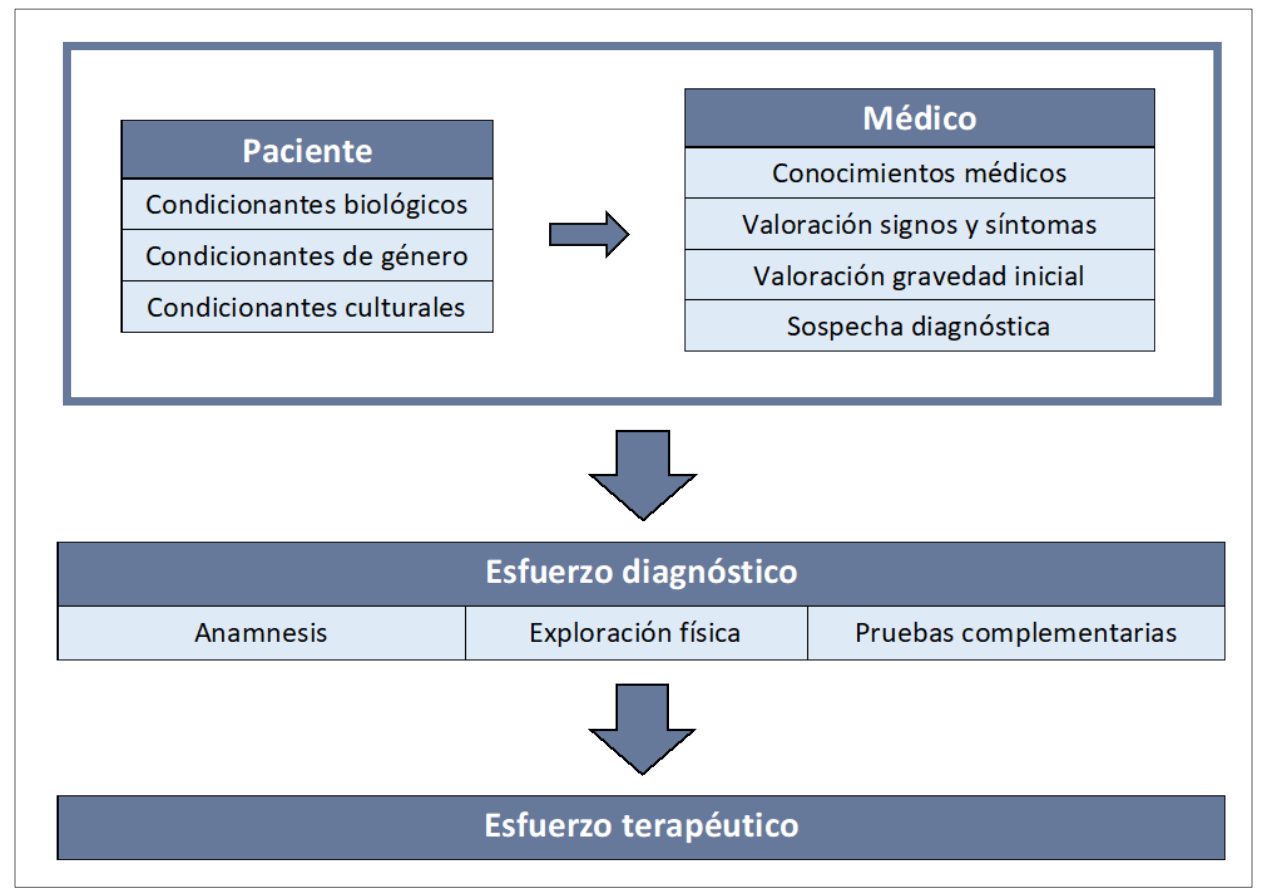


Por otro lado, los médicos tienen un conocimiento fruto de la evidencia científica e interpretan y valoran los signos y síntomas, llegando a una sospecha diagnóstica y a una valoración de la gravedad del problema padecido y, en consecuencia, a un determinado esfuerzo diagnóstico, basándose en la anamnesis, la exploración clínica y las pruebas complementarias.

En esta fase, hay que tener en cuenta que:

- Hay diferentes interpretaciones por parte de los médicos que pueden dar lugar a diferentes versiones de los mismos síntomas y signos. Concretamente, se pueden atribuir diferentes significados al cuadro clínico explicado, según sean presentados por pacientes hombres o mujeres, cosa que supondría un sesgo de género en el diagnóstico.

- La historia explicada por los pacientes no es idéntica a la historia clínica, aunque pueden ser compatibles. Por lo tanto, es la traducción que se hace después de escuchar la historia de quien lo padece, teniendo diversas lecturas según quien lo escuche.

- La capacidad de observación del médico está limitada por su experiencia personal/profesional y determina la perspectiva desde la cual se observa, por la cual cosa impactará en el que puede ver y no ver.

Hasta la actualidad, la mayoría de las investigaciones sobre sesgos de género en el esfuerzo diagnóstico están focalizadas en la mayor o menor realización de pruebas diagnósticas. Por lo tanto, al partir estos estudios de la prueba diagnóstica y verificar si tienen o no la patología en cuestión según sexo, es posible que ciertos síntomas atípicos de esta patología no se hayan considerado y aquellos pacientes, que en la mayoría son mujeres, hayan quedado excluidas del sistema sanitario, por la cual cosa el sesgo de género no se detectaría, ya que solo serán estudiadas aquellas personas que lleguen al esfuerzo diagnóstico. Además, se debe considerar que la mayoría de los diagnósticos, en especial en atención primaria, se realizan mediante anamnesis y examen clínico, y no mediante pruebas diagnósticas.

Hay que tener en cuenta que el sesgo de género en el esfuerzo diagnóstico condiciona el esfuerzo terapéutico, ya que la probabilidad de que quien padece sea tratado es casi nula si, por cualquier causa, queda excluido del proceso diagnóstico, o disminuye si en el proceso diagnóstico no se realizan las pruebas oportunas. 


\subsection{En el esfuerzo terapéutico}

El esfuerzo terapéutico depende del esfuerzo diagnóstico, del diagnóstico en sí y de otros determinantes propios de la relación médico-paciente (Ruiz Cantero, 2009).

Se dispone de evidencia que demuestra que no únicamente el género del paciente es relevante, sino que el género del profesional interviene de manera decisiva. Este aspecto es estudiado notablemente respecto a los estilos de comunicación y la relación profesional-paciente, de manera que el marco relacional en el cual se desarrolla el acto médico es diferente en función de si paciente y profesional son del mismo o de diferentes géneros.

Tal y como se muestra en Tasa-Vinyals et al. (2015), la existencia de sesgo de género ha sido descrita de manera consistente en una gran variedad de contextos geográficos y situaciones clínicas, de entre los que destacan:

\section{Frecuencia y tipo de uso de los servicios de hospital y ambulatorio}

Los hombres utilizan más los servicios sanitarios especializados y/o urgentes (hospitalarios), mientras que las mujeres utilizan más la atención primaria. Este último hecho puede ser explicado por una menor derivación de las mujeres a la asistencia hospitalaria (Ruiz Cantero, 2009). Esta utilización diferencial es responsabilidad de los pacientes por una parte, pero también del médico y del sistema sanitario (Ruiz-Cantero y Verdú-Delgado, 2004).

\section{Aplicación de métodos preventivos, diagnósticos y terapéuticos}

En numerosos ámbitos se ha observado que uno de los géneros tiende a acumular el uso de los recursos preventivos, diagnósticos y terapéuticos (y del coste correspondiente), aunque el problema de salud en cuestión se presente de manera comparable en ambos géneros, o incluso sea más frecuente en el género menos tratado (Ruiz-Cantero y VerdúDelgado, 2004).

\section{Demora y espera de la asistencia sanitaria}

En general se ha visto que, a igual necesidad o problema de salud, las mujeres tardan más en buscar ayuda médica y, además, también tardan más en ser atendidas una vez la buscan. El rol de género femenino puede condicionar esta demora en la búsqueda de asistencia, ya que el papel de cuidadora lo ejerce la mujer mayoritariamente (RuizCantero y Verdú-Delgado, 2004). 


\section{Prescripción y consumo de fármacos. Medicalización del malestar emocional femenino}

Entre las mujeres hay, en general, más consumo de fármacos psicotrópicos que entre los hombres. Esto puede ser dado por una mayor prevalencia de depresión y ansiedad o porque, al quejarse, inducen más la prescripción que los hombres. Pero también puede deberse a que los médicos atribuyan a factores psicológicos, más fácilmente en mujeres que en hombres, los síntomas físicos o tiendan a prescribir fármacos por síntomas depresivos de baja intensidad a mujeres más que a hombres. Por lo tanto, el malestar emocional de las mujeres está medicalizado, habitualmente, con estrategias terapéuticas sintomáticas erróneas y ciegas a las causas (Ruiz-Cantero y Verdú-Delgado, 2004; Ruiz Cantero, 2009).

\section{Problemas de salud propios de las mujeres. Medicalización de la fisiología femenina}

Hay problemas de salud más frecuentes o propios de las mujeres. Se ha descrito un sobreuso o uso dudoso de acuerdo con la evidencia científica disponible de ciertas prestaciones sanitarias, así como de la terapia hormonal substitutiva, donde se medicaliza un proceso natural como la menopausia (Ruiz-Cantero y Verdú-Delgado, 2004).

El enfoque centrado en la biología reproductiva de las mujeres ha llevado a que la salud del sexo femenino se trate de manera equivalente a la salud reproductiva, dando lugar a un interés inferior en los problemas de salud no reproductiva (Ruiz Cantero, 2009).

\section{Consecuencias del sesgo de género}

Hay importantes consecuencias del sesgo de género en la salud de las mujeres. Algunas que se encuentran relacionadas con el conocimiento médico, el manejo clínico y la prestación de la asistencia sanitaria se exponen a continuación (Ruiz y Verbrugge, 1997):

- La asunción de similitudes entre géneros en la experiencia de enfermedades lleva a que la mayoría de la investigación sea sobre enfermedades crónicas fatales, dando lugar a una falta de avances en el manejo de las condiciones crónicas no fatales, las cuales son más frecuentes en mujeres.

- La invisibilización de las mujeres bajo la normatividad masculina conduce a una infrareportación de las posibles diferencias existentes en la manera de enfermar. Esto lleva a una mayor proporción de mujeres que de hombres con diagnóstico de «síntomas y signos no específicos» tanto en los registros del servicio de salud como en los certificados de defunción. Los pacientes clasificados como síntomas 
y signos no específicos pueden padecer una enfermedad identificable, ya sea en una etapa temprana no capturada por criterios diagnósticos contemporáneos o por un conjunto atípico (no masculino) de quejas por la enfermedad. La ausencia de tratamiento o un tratamiento inadecuado en este punto puede conducir al empeoramiento de la enfermedad.

- La asunción de diferencias de género da lugar a una prestación diferencial de servicios sanitarios y resultados de tratamiento. Estas presunciones iniciales de que los hombres y las mujeres difieren en las percepciones de los síntomas y la gravedad de la enfermedad pueden ser incorrectas e influir mucho en las decisiones de tratamiento y resultados.

La falta de investigación sensible a los fenómenos de género contribuye a la perpetuación y a la legitimación por omisión de las creencias y actitudes de género predominantes en la sociedad, de las cuales los profesionales sanitarios difícilmente pueden escapar sin formación específica en términos de género (Tasa-Vinyals, Mora Giral y Raich, 2015).

Debido a que la mayoría de los estudios en los cuales se basa la práctica médica se realizan predominantemente con sujetos hombres o animales machos, el sesgo de género tiene más probabilidades de perjudicar a las pacientes mujeres por un mecanismo de desconocimiento de sus formas de enfermar y de curarse, la cual cosa no quiere decir que no pueda perjudicar a los pacientes varones, sea directamente o indirectamente (Ruiz y Verbrugge, 1997). De hecho, la inequidad de género perjudica tanto a las mujeres como a los hombres (Ruiz-Cantero et al., 2007).

\subsection{Limitaciones y barreras actuales}

En la práctica clínica, el sesgo de género se produce en los servicios de salud a causa de una estratificación política más amplia de la sociedad en relación con el género. No es deliberada (si es así, podría interpretarse como una mala praxis). Los médicos no suelen creer que el sesgo de género existe, basándose en sus prácticas clínicas bien motivadas y entrenadas (Ruiz y Verbrugge, 1997). Para llegar a una consciencia de género que lleve a cambios en este ámbito, haría falta implementar cuestiones de género en la educación médica. Esto genera retos específicos, ya que se observa una resistencia institucional al cambio, incerteza sobre qué cuestiones de género se deberían incluir en un plan de estudios y una falta de directrices prácticas para la implementación (Henrich, 2004). 
En el ámbito de la investigación, las discusiones científicas sobre el sesgo de género tienden a caer en dos posiciones: existe o no. La verdad es ciertamente diversa: el sesgo de género existe en algunos ámbitos y en otros no (Ruiz y Verbrugge, 1997). Además, a pesar de los avances, persisten carencias conceptuales y metodológicas que limitan el uso de datos segregados por sexo o género (Cueva Beteta, 2006). El impacto de las medidas gubernamentales en la promoción de la equidad de género en la investigación ha demostrado ser ineficaz, tanto en la participación de las mujeres en los estudios como en el análisis de resultados (Ramasubbu, Gurm y Litaker, 2001).

\subsection{Evolución y perspectivas de futuro}

Tal y como se menciona en Hamberg (2008), el sesgo de género en la asistencia sanitaria continuará siendo un campo de investigación importante durante los próximos años. Aún existe la necesidad de realizar estudios descriptivos sobre las disparidades de género en muchas especialidades, trastornos y países. También es preciso aprender más sobre los procesos cognitivos y de interacción que conducen al sesgo de género en el trabajo clínico, y el sesgo de género que se construye en diseños y análisis de investigación. Por lo tanto, se deben tener en cuenta diferentes ámbitos que se tratan a continuación.

\section{La investigación}

La perspectiva de género debe estar presente en todas las etapas de la investigación, desde la observación hasta la preparación de la teoría científica, pasando por la elaboración de la hipótesis, la experimentación y la demostración o la refutación de las hipótesis (Aldasoro, 2017).

El número de hombres y mujeres incluidos en los estudios debe ser suficiente para permitir análisis de sexo y de género y evaluar si el sexo, el género o ambos son importantes para los resultados (Ruiz y Verbrugge, 1997). Por ello, se necesita un mayor soporte por parte de las agencias de financiación, y estas tendrían que desarrollar requisitos para la inclusión de animales hembras en ciencias básicas y de mujeres en estudios translacionales y ensayos clínicos (Miller, 2014). Esto permitiría generar un adecuado conocimiento científico sobre las mujeres en un futuro, haciendo que no sea necesario transferir el conocimiento sobre los hombres a las directrices para las mujeres (Hamberg, 2008).

Además, un mejor equilibrio y reorientación de la investigación patología-específica son necesarios para que condiciones que predominan en las mujeres, como aquellas no 
fatales, reciban una atención adecuada. La investigación sobre estas condiciones también beneficiará los hombres que, si viven suficiente, finalmente adquieren el mismo repertorio de problemas no fatales. Por lo tanto, hace falta que las mujeres sean incluidas en estudios de enfermedades fatales y también hay que asegurar la inclusión de hombres en estudios de investigación sobre las no fatales (Ruiz y Verbrugge, 1997).

También es necesaria una mayor investigación sobre la ceguera de género y las concepciones previas sobre el género en conceptos médicos básicos y definiciones que se dan por hecho. Hacen falta diseños y modelos analíticos nuevos y fiables en la búsqueda de diferencias biológicas, diseños y modelos que integren y consideren el impacto de las condiciones socioculturales en los resultados (Hamberg, 2008).

\section{La difusión del conocimiento}

Desde las revistas médicas, se debe instaurar explícitamente una política para promover la igualdad de género en la publicación científica, tal y como recomienda la Asociación Europea de Editores de Ciencia y como han hecho otras revistas, por ejemplo, The Lancet y Nature. Esta debe incluir recomendaciones sobre cómo tener en cuenta el género en la publicación científica y acciones de promoción de la igualdad de género en la gestión de la revista (Borrell et al., 2015).

Desde el punto de vista de la publicidad farmacéutica, la precisión de la transferencia de conocimiento es esencial para evitar el sesgo de género en la práctica médica y para conseguir una prescripción de medicamentos de calidad según evidencias basadas en el conocimiento (Johnson, Greaves y Repta, 2009).

\section{La práctica clínica}

Se debe potenciar la generación y aplicación en la práctica clínica de pautas que tengan en cuenta sistemáticamente el sexo biológico de cada paciente, además de otros factores que se entrecruzan con este como el género, la edad, la raza y el estado socioeconómico (Tannenbaum, Norris y McMurtry, 2019). A causa del aumento en la última década de publicaciones de investigación médica que consideran los participantes masculinos y femeninos por separado y un aumento del número de revisiones sistemáticas que disgregan los resultados según el sexo, las evidencias sobre las cuales se deberían basar las recomendaciones de directrices específicas por sexo son cada vez más accesibles a los desarrolladores de pautas (Tannenbaum, Norris y McMurtry, 2019). 
La incorporación de la perspectiva de género en los planes estratégicos de salud está erigiéndose como necesaria en los últimos años, y algunos trabajos concluyen que es cada vez más notoria en las actuaciones sanitarias de las administraciones estatales y autonómicas de España. También las guías clínicas y los manuales diagnósticoterapéuticos están incorporando progresivamente la perspectiva de género como medida clave para aumentar su validez y vigencia (Tasa-Vinyals, Mora Giral y Raich, 2015).

La implementación de diagnósticos y tratamientos equitativos de género requiere tiempo, compromiso y soporte institucional y político. La traducción a la práctica de los resultados disponibles de la investigación puede tardar entre cinco y quince años, sobre todo si se tiene en cuenta el desarrollo de nuevos fármacos. Esto requiere de una acción inmediata, ya que éticamente no pueden ignorarse estos resultados sustentados en la investigación (Regitz-Zagrosek, 2012).

\section{La institucionalización de la perspectiva de género en las ciencias médicas}

Mejorar el enfoque educativo es fundamental para disminuir el desconocimiento y/o la actitud indiferente de profesionales e instituciones respecto al sesgo de género (TasaVinyals, Mora Giral y Raich, 2015). La implementación de la educación sobre procesos, reacciones y tratamientos relacionados con el sexo y el género en los planes de estudios de la escuela médica y otras formas de educación sanitaria es un paso importante en la concienciación sobre el sesgo de género. Sin embargo, el conocimiento sobre la perspectiva de género en la educación de los estudiantes de medicina es escaso y se necesita más investigación para dilucidar la mejor manera de implementarlo (Hamberg, 2008).

El soporte institucional es imprescindible en el ámbito organizativo para asegurar la sostenibilidad de la perspectiva de género, y se tienen que desarrollar guías nacionales para su inclusión en los diferentes niveles educativos (Ruiz-Cantero et al., 2019).

\subsection{Limitaciones del estudio}

Cabe destacar que en esta revisión bibliográfica no se ha tratado la totalidad de las disciplinas de las ciencias de la salud que se pueden ver afectadas por el sesgo de género a causa de su gran amplitud. No se han incluido en la revisión los artículos centrados en cómo afecta el sesgo de género en la relación entre profesionales de la salud ni se han introducido aquellos que hacen un abordaje profundo en el ámbito educativo y formativo. 
Los temas tratados han sido enfocados desde una perspectiva de género binaria por falta de bibliografía existente sobre los efectos del sesgo de género en personas transgénero o intersexuales.

Respecto a las publicaciones sobre el tema, el número es limitado, aunque está en aumento en relación con el creciente interés social y científico por las diferencias y desigualdades de género (Ruiz y Verbrugge, 1997). A pesar de que cada vez hay más investigación sobre la carga sanitaria y las necesidades de las minorías de género, las cuestiones transgénero continúan siendo marginadas (Reisner et al., 2016).

\section{CONCLUSIONES}

Actualmente se encuentran limitaciones relacionadas con el sesgo de género en el ámbito de la investigación, la difusión de conocimientos y la práctica clínica. Por ello, el sesgo de género continuará siendo un campo de investigación importante durante los próximos años, con el objetivo de seguir avanzando en las áreas previamente mencionadas y en la institucionalización de la perspectiva de género en las ciencias médicas.

La incorporación de la perspectiva de género en la asistencia sanitaria es un proceso que aún requiere un aumento de la investigación y del conocimiento. También son necesarias la implementación de múltiples cambios y adaptaciones en diversos ámbitos y la coordinación entre un gran número de entidades dentro de la comunidad científica. Por lo tanto, se trata de un objetivo de elevada dificultad.

Con todo, es de gran interés intentar integrarla en la práctica clínica para poder identificar posibles sesgos de género, ya que la medicina de género es un elemento importante a tener en cuenta dentro de la medicina personalizada y la atención centrada en el paciente, cuyo desarrollo es esencial para alcanzar el objetivo estándar de un enfoque holístico de los pacientes y las enfermedades. 


\section{BIBLIOGRAFÍA}

ALDASORO, E. Reflexiones acerca de las dificultades en la integración del enfoque de género en la inestigación en salud. En: Inguruak. 2017, vol. 44, pp. 263-271.

Borrell, C. et al. Las desigualdades de género en la ciencia: Gaceta Sanitaria da un paso adelante. En: Gaceta Sanitaria. 2015, vol. 29, núm. 3, pp. 161-163.

Cambronero Saiz, B.; Ruiz Cantero, M. T.; Papí Gálvez, N. Quality of pharmaceutical advertising and gender bias in medical journals (1998-2008): A review of the scientific literature. En: Gaceta Sanitaria. 2012, vol. 26, núm. 5, pp. 469-476.

Chilet-Rosell, E. Gender bias in clinical research, pharmaceutical marketing, and the prescription of drugs. En: Global Health Action. 2014, vol. 7, núm. 1.

CIHR - INSTITUTE OF GENDER AND HEALTH. What a difference sex and gender make. 2012. Disponible en: https://cihr-irsc.gc.ca/e/44734.html.

COMISION EuRopeA. 100 palabras para la igualdad. Glosario de términos relativos a la igualdad entre mujeres y hombres. 1998.

Cueva Beteta, H. What is missing in measures of Women's Empowerment? En: Journal of Human Development. 2006, vol. 7, núm. 2, pp. 221-241.

Esteban Galarza, M. El estudio de la salud y el género: las ventajas de un enfoque antropológico y feminista. En: Salud Colectiva. 2006, vol. 2, núm. 1, pp. 9-20.

HAMBERG, K. Gender bias in medicine. En: Women's Health. 2008, vol. 4, num. 3, pp. $237-$ 243.

HEIDARI, S. et al. Sex and Gender Equity in Research: rationale for the SAGER guidelines and recommended use. En: Research Integrity and Peer Review. 2016, vol. 1, núm. 2.

HenRICH, J. B. Women's Health Education Initiatives: Why Have They Stalled? En: Academic Medicine. 2004, vol. 79, núm. 4, pp. 283-288.

Hoffmann, D.; Tarzian, A. The Girl Who Cried Pain: A Bias Against Women in the Treatment of Pain. En: Journal of Law, Medicine \& Ethics. 2001, vol. 29, pp. 13-27.

JoHnSON, J. L.; GREAVES, L.; REPTA, R. Better science with sex and gender: Facilitating the use of a sex and gender-based analysis in health research. En: International Journal for Equity in Health. 2009, vol. 8, núm. 14, pp. 1-11.

MAUVAIS-JARVIS, F. et al. Sex and gender: modifiers of health, disease, and medicine. En: The Lancet. 2020, vol. 396, pp. 565-582.

MiLLER, V. M. Why are sex and gender important to basic physiology and translational and individualized medicine? En: American Journal of Physiology - Heart and Circulatory Physiology. 2014, vol. 306, pp. 781-788.

RAMAsubBu, K.; GuRM, H.; LiTAKer, D. Gender bias in clinical trials: Do double standards still apply? En: Journal of Women's Health and Gender-Based Medicine. 2001, vol. 10, núm. 8, pp. 757-764.

Regitz-Zagrosek, V. Sex and gender differences in health. Science \& Society Series on Sex and Science. En: EMBO reports. 2012, vol. 13, núm. 7, pp. 596-603. 
REISNER, S. L. et al. Global health burden and needs of transgender populations: a review. En: The Lancet. 2016, vol. 388, pp. 412-436.

RisberG, G. et al. Gender in medicine - An issue for women only? A survey of physician teachers' gender attitudes. En: International Journal for Equity in Health. 2003, vol. 2, núm. 10.

Risberg, G.; Johansson, E. E.; HAMBerg, K. A theoretical model for analysing gender bias in medicine. En: International Journal for Equity in Health. 2009, vol. 8, núm. 28.

Rosser, S. V. Clinical Trials and Experimental Science, Bias Against Women in. En: The Wiley Blackwell Encyclopedia of Gender and Sexuality Studies. 2015.

Ruiz, M. T.; Verbrugge, L. M. A two way view of gender bias in medicine. En: Journal of Epidemiology and Community Health. 1997, vol. 51, pp. 106-109.

Ruiz CANTERo, M. T. Sesgos de género en la atención sanitaria. Granda: Escuela Andaluza de Salud Pública, 2009.

Ruiz-CANTERo, M. T.; Verdú-Delgado, M. Sesgo de género en el esfuerzo terapéutico. En: Gaceta Sanitaria. 2004, vol. 18, supl. 1, pp. 118-125.

Ruiz-CANTERO, M. T. et al. A framework to analyse gender bias in epidemiological research. En: Journal of Epidemiology and Community Health. 2007, vol. 61, núm. 2, pp. 46-53.

Ruiz-CANTERo, M. T. et al. Gender agenda in health sciences education: international experiences to reduce time in Spain. En: Gaceta Sanitaria. 2019, vol. 33, núm. 5, pp. 485-490.

SHANNON, G. et al. Gender equality in science, medicine, and global health: where are we at and why does it matter? En: The Lancet. 2019, vol. 393, pp. 560-569.

SIMON, V. Wanted: Women in clinical trials. En: Science, 2005, vol. 308, pp. 1517.

Tannenbaum, C.; Norris, C. M.; McMurtry, M. S. Sex-Specific Considerations in Guidelines Generation and Application. En: Canadian Journal of Cardiology. 2019, vol. 35, pp. 598-605.

Tasa-Vinyals, E.; Mora Giral, M.; Raich, R. Sesgo de género en medicina: concepto y estado de la cuestión. En: Cuadernos de medicina psicosomática y psiquiatria de enlace. 2015, vol. 113, pp. 14-25.

VALls-Llobet, C. Desigualdades de género en salud pública. En: Quadern CAPS. 2001, vol. 30, pp. 34-40.

VÁzquez-Santiago, S.; Garrido Peña, F. El enfoque de género en las necesidades de atención sociosanitaria. En: Enfermería Clínica. 2016, vol. 26, núm. 1, pp. 76-80. 